\title{
Determination of Total Plutonium Content in Spent Nuclear Fuel Assemblies with the Differential Die-Away Self-Interrogation Instrument
}

\author{
Alexis C. Kaplan ${ }^{1,2}$, Vladimir Henzl ${ }^{1}$, Howard O. Menlove ${ }^{1}$, Martyn T. Swinhoe ${ }^{1}$, Anthony P. Belian ${ }^{1}$, \\ Marek Flaska ${ }^{2}$, Sara A. Pozzi ${ }^{2}$ \\ 1. Los Alamos National Laboratory, P. O. Box 1663, Los Alamos, NM 87544, USA \\ 2. Department of Nuclear Engineering and Radiological Sciences, University of Michigan, \\ 500 S State St, Ann Arbor, MI 48109, USA
}

\begin{abstract}
As a part of the Next Generation Safeguards Initiative Spent Fuel project, we simulate the response of the Differential Die-away Self-Interrogation (DDSI) instrument to determine total elemental plutonium content in an assayed spent nuclear fuel assembly (SFA). We apply recently developed concepts that relate total plutonium mass with SFA multiplication and passive neutron count rate. In this work, the multiplication of the SFA is determined from the die-away time in the early time domain of the Rossi-Alpha distributions measured directly by the DDSI instrument. We utilize MCNP to test the method against 44 pressurized water reactor SFAs from a simulated spent fuel library with a wide dynamic range of characteristic parameters such as initial enrichment, burnup, and cooling time. Under ideal conditions, discounting possible errors of a real world measurement, a root mean square agreement between true and determined total Pu mass of $2.1 \%$ is achieved.
\end{abstract}

(1)

\section{INTRODUCTION}

The Differential Die-Away Self-Interrogation (DDSI) technique for nondestructive spent fuel assay is investigated as a part of the Next Generation Safeguards Initiative Spent Fuel project (NGSI-SF) [1]. DDSI is a passive neutron coincidence counting technique that utilizes neutrons primarily from spontaneous fission (SF) in the assayed spent fuel assembly (SFA) to preferentially interrogate its fissile material content [2]. Data are collected in list-mode in order to construct and utilize a Rossi-alpha distribution (RAD) for SFA characterization. In our previous work, we have demonstrated by means of high fidelity simulations the ability of the DDSI instrument to evaluate SFA multiplication with high accuracy; i.e., within $\sim 0.8 \%$ [3].

Applying a recently developed concept that correlates SFA multiplication $(M)$ and passive neutron count rate $(P N)$ with total elemental plutonium content $\left(m_{P u}\right)$ in the SFA [4], we demonstrate that $m_{P u}$ can be determined in simulation space using the DDSI instrument with a similarly high accuracy. The method has been tested against 44 simulated SFAs from Spent Fuel Library 2a (SFL2a), which is an assortment of simulated SFAs with varying burnup (BU), initial enrichment (IE), and cooling time (CT) [5]. In these simulations, $m_{P u}$ was determined with root mean square (RMS) error of $2.1 \%$ with respect to its true content as defined in the simulation input files. The current results are qualitatively as well as quantitatively nearly identical to those obtained previously for the Differential Die-Away instrument that is based on an active neutron interrogation technique with an external pulsed neutron generator and tested against Spent Fuel Library-1 [6], thus demonstrating the robustness of the method. This apparent robustness in simulation space makes it a promising technique for experimental verification which will be 
the ultimate test of the method. Within this work, we limit ourselves to discussing the theory of the DDSI instrument and total $\mathrm{Pu}$ determination and present simulation results for determining plutonium content in SFL2a assemblies.

\section{DDSI INSTRUMENT AND RESPONSE}

The simulated DDSI instrument consists of four modules each with $14{ }^{3} \mathrm{He}$ detectors embedded in high-density polyethylene. The detectors have a $2.54 \mathrm{~cm}$ diameter, $40.0 \mathrm{~cm}$ active length, and $6 \mathrm{~atm}$ pressure. A cross-sectional view of the simulated instrument is shown in Fig. 1.

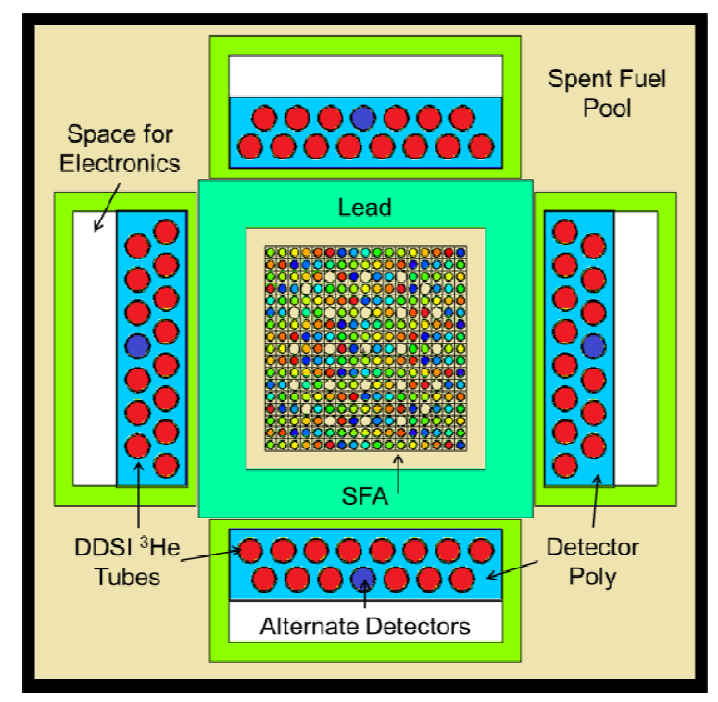

Fig. 1. Cross-sectional view of the simulated DDSI instrument.

During the envisioned assay, the SFA is lowered into the middle of the instrument inside the spent fuel pool and data are collected in list-mode, resulting in the time of capture of every neutron in any of the 56 detectors being recorded. Spontaneous fission is abundant in SFAs, primarily from the presence of ${ }^{244} \mathrm{Cm}$. However, in the case of SFAs with high CT and low BU, spontaneous fission of ${ }^{240} \mathrm{Pu}$ may be the most prominent contributor together with neutrons from $(\alpha, n)$ reactions initiated mostly by significant presence of ${ }^{241} \mathrm{Am}$ as quantified for SFL-1 in detail in [4]. As the assay is conducted in water, the SF and $(\alpha, n)$ neutrons thermalize and re-enter the fuel pins to induce additional fissions preferentially on the fissile isotopes such as ${ }^{235} \mathrm{U},{ }^{239} \mathrm{Pu}$ and ${ }^{241} \mathrm{Pu}$. Depending on the multiplication of the SFA, which reflects its isotopic composition, fission chains of various length and multiplicity will develop. While the rise and fall, i.e., die-away, of the neutron subpopulation in the fission chain induced by any individual SF or $(\alpha, n)$ reaction cannot be measured on a case by case basis, various aspects of the evolution of the entire neutron population can be determined utilizing list-mode based neutron coincidence counting techniques. Each detected neutron in the list-mode data is treated as a trigger and the time until the detection of subsequent neutrons within a pre-determined time window (typically $\sim 1000 \mu \mathrm{s}$ ) is recorded. The RAD is a histogram of the neutron detection times after a triggering event. This way a RAD can be constructed which allows us to quantify real and accidental coincidences among neutrons as well as to observe the die-away (and its possible dynamic evolution) of the neutron population. In the context of traditional neutron coincidence measurements the die-away time describes the decrease of the neutron population over time and is typically represented by a single exponential of the form: 


$$
N(t)=N(0) e^{-t / \tau}
$$

72 where $N(t)$ is the neutron population at time $t, N(0)$ is the initial population, and $\tau$ is the mean neutron 73 lifetime in the system, i.e., the die-away time [7]. However, due to re-introduction of thermalized 74 neutrons to the SFA, which is typically undesirable and absent in traditional neutron coincidence counting 75 approaches, the real correlation between two neutrons exhibits two distinctive modes. The first mode 76 fast correlation - is a result of correlations between neutrons from the same fission event, be it 77 spontaneous or induced fission (IF), or from fission chains where the inducing neutrons have not been 78 reduced to thermal energies. The fast correlation reflects the mean lifetime of a single neutron in the 79 system, and is on the order of $19 \mu \mathrm{s}$, which is the characteristic die-away time of the detection system. 80 The second mode - slow correlation - is a result of correlations among neutrons from different fission 81 events but within the same fission chain that have undergone the thermal-neutron scattering time-delay. 82 The slow correlation reflects the difference in detection times between two neutrons of a fission chain 83 separated possibly by tens or even hundreds of $\mu$ s due to the time required for a neutron from one fission 84 event to thermalize in the water, re-enter a fuel pin and induce another fission event. As a consequence 85 the RAD reflects both of these correlations, each with a significantly different characteristic time 86 constant. This prevents any accurate description of the system by eq. (1). However, the sum of two 87 exponentials provides a sufficiently accurate description of the RAD obtained from the simulated response of the DDSI instrument when assaying SFAs:

$$
N(t)=N_{\text {fast }} \cdot e^{-t / \tau_{\text {fast }}}+N_{\text {slow }} \cdot e^{-t / \tau_{\text {slow }}}
$$

where $N(t)$ is the neutron population at time $t, N_{\text {fast }}$ is the initial population which will be detected in the fast correlation mode and $N_{\text {slow }}$ the initial population that will be detected in slow correlation mode. Thus, $\tau_{\text {fast }}$ represents the mean lifetime (i.e., die-away time) of an individual detected neutron in the system, while $\tau_{\text {slow }}$ represents the mean lifetime of the entire neutron subpopulation that consists of multiple neutron generations. The interplay of these two quantities is in the early die-away time, $\tau_{\mathrm{e}}$ defined as the die-away time of a single exponential fit of the early time domain of the RAD from 5-52 $\mu$ s [3]. Figure 2 is an example of a RAD constructed from the simulated DDSI instrument response with the fast and slow components as well as the early die-away time displayed and accidental coincidences excluded. 


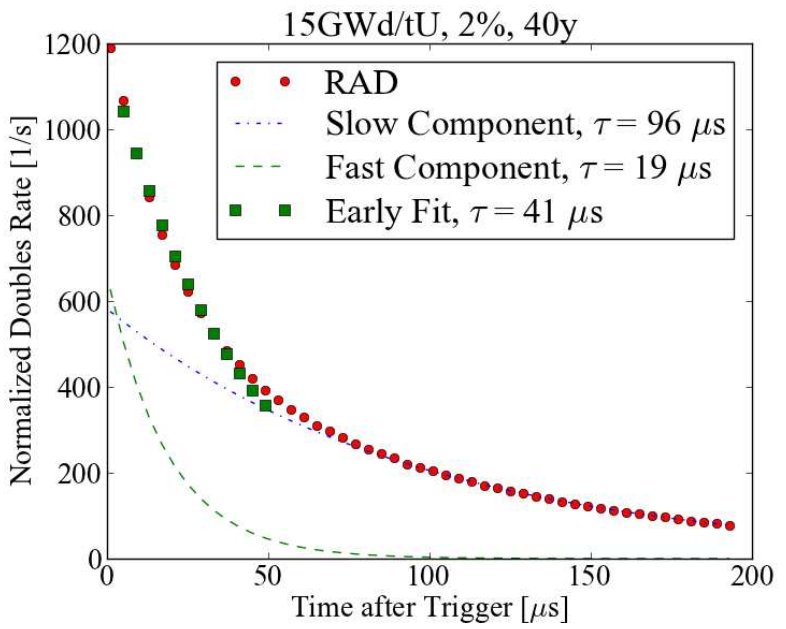

100

101

102

103

104

105

106

107

108

109

110

111

112

113

114

115

116

Fig. 2. An example of RAD from simulated assay of SFA with $B U$ of $15 \mathrm{GWd/tU}, 2 \%$ IE, and 40 y CT. The two additive single exponentials - the fast and slow component - are also displayed. The early die-away time is shown with squares.

\section{DETERMINATION OF THE TOTAL PLUTONIUM CONTENT}

In the work of Henzl et al. [4], the total plutonium content within an assembly is found to be related to the SFA multiplication and the detected total neutron count rate provided the CT is known or constant within the ensemble of the SFAs that are being assayed (e.g. all SFAs from the same reactor discharge). The multiplication reflects the competition between the fissile content and the amount and composition of the neutron absorbers (fission products and minor actinides), and is implicitly defined by the SFA characteristic parameters such as IE, BU, and CT. The total neutron emission, however, reflects mass content of major spontaneous fission isotopes in the assembly including ${ }^{242} \mathrm{Cm},{ }^{244} \mathrm{Cm}$, and ${ }^{240} \mathrm{Pu}$ as well as the main contributors to neutron production via $(\alpha, n)$ reaction (e.g. $\left.{ }^{241} \mathrm{Am}\right)$. Since the majority of these isotopes are produced by processes that include one or more neutron captures on individual Pu isotopes, their quantity, and thus the neutron emission rate, is closely correlated to the total content of elemental plutonium (i.e., $m_{P u}$ ) [8]. A visual representation of various production modes of individual actinides in the process of nuclear burning is displayed in Fig.3. 


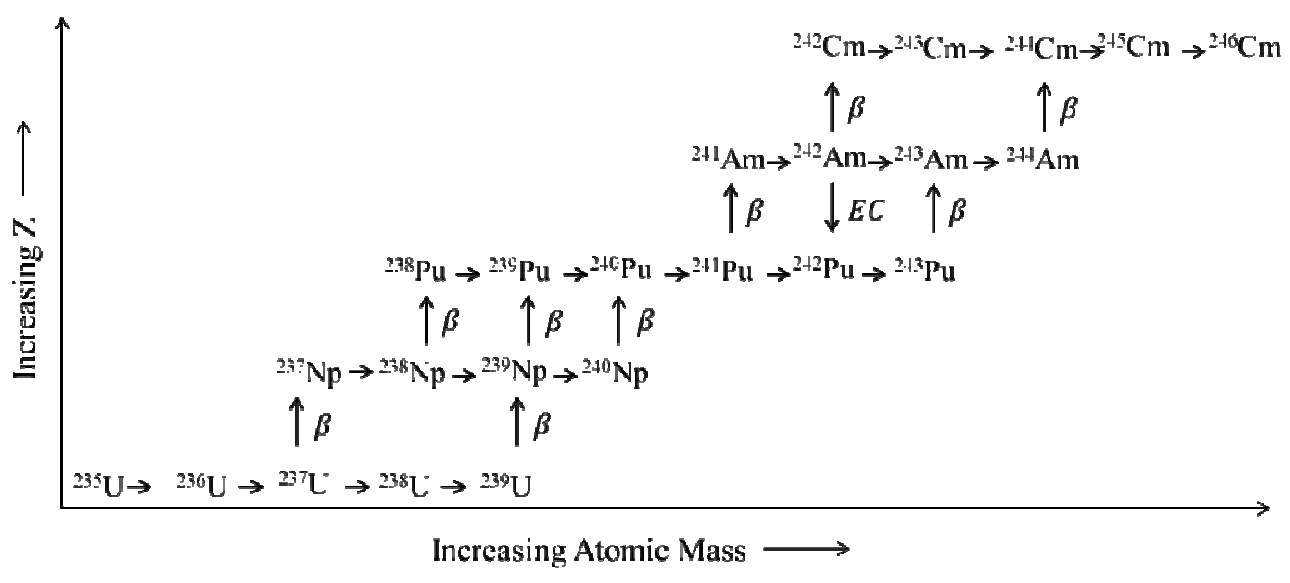

Fig. 3. Visual representation of the most significant actinide production modes in the spent nuclear fuel. (Note: The alpha decay is omitted in order to focus on production of the heaviest actinides)

In this work, we ultimately utilize eq.(16) from [4] relating the neutron emission rate $(N E)$, multiplication $(M)$, and total plutonium mass $\left(m_{P u}\right)$

$$
m_{p u}=a(C T)\left(\frac{M+2 c}{M+c}\right)\left[\left(\frac{M}{M+2 c}\right) N E\right]^{b(C T)}
$$

with $a, b$, and $c$ being instrument dependent calibration parameters, which in the case of $a$ and $b$ may also be functions of CT. However, as the authors of [4] modified the equation above to include quantities measurable by the DDA instrument (eq.(20) in [4]), we modify the equation to include only quantities directly measurable by the DDSI instrument. Previously [3] we reported our finding that the die-away time of the early time domain (5-52 $\mu \mathrm{s})$ of the RAD of the DDSI instruments response is quadratically related with the SFA multiplication:

$$
M=a_{1} \tau_{e}^{2}+a_{2} \tau_{e}+a_{3}
$$

where $a_{1}, a_{2}$, and $a_{3}$ are the fitting (i.e., calibration) parameters. In the case of the DDSI instrument the singles rate $(S)$ is identical to the passive neutron count rate used in [4], and through a simple relation with $M$ it is then directly representative of the NE term in eq.(3):

$$
N E=S /(M \times \varepsilon)
$$

where $\varepsilon$ is the system efficiency. Assuming that efficiency is absorbed into the calibration parameters, the eq.(3), i.e., the expression for determination of $m_{P u}$, can be rewritten using only quantities directly measurable by the DDSI instrument as follows:

$$
m_{p u}=d(C T)\left(\frac{\tau_{e}+g}{\tau_{e}+h}\right)\left(\frac{S}{\tau_{e}+g}\right)^{f(C T)}
$$

where $d, f, g$ and $h$ are the calibration parameters which depend on the instrument design and in case of $d$ and $f$ also on CT. 


\section{RESULTS}

All simulations were conducted with $\mathrm{MCNPX}^{\mathrm{TM}}$ v2.7.0 [9]. Coincidence counting data are simulated in the form of RADs which are discussed in detail in [3]. SFAs are defined on a pin-by-pin basis from material definitions developed as part of NGSI-SF project [1]. In total, we simulated the assay of 44 pressurized water reactor assemblies from SFL2a with IE varying from 2 to $5 \%$, BU varying from 15 to $60 \mathrm{GWd} / \mathrm{tU}$, and CT varying from 5 to 80 years. The SFAs are $17 \times 17$ Westinghouse type with one radial region tracked through a 1/8 core using the code Monteburns to track isotopic changes through the burning process [10]. While the SFLs are designed to describe the complex isotopic composition of SFAs as realistically as possible, various approximations are still being made during their development by assuming, for example, homogenous axial BU profiles and disregarding fuel expansion and rim effects, among others.

The two panels of Fig. 4 display results of the simulations in terms of the detected singles rate $(S$; left panel) and the measured die-away time $\left(\tau_{e}\right)$ in the early time domain (4-52 $\mu \mathrm{s}$; right panel). Both panels thus demonstrate the complex interplay of IE, BU and CT on the measurable signals with respect to the true $\mathrm{Pu}$ content, yet the correction introduced in eq. (6) removes most of these dependences without an explicit knowledge of IE, BU and possibly even CT, as can be seen in left panel of Fig. 5. The right panel of Fig. 5 then displays the relative differences between the $m_{P u}$ determined from eq. (6) and the true $m_{P u}$ as known from the material definition of individual SFAs. The numerical summary of data displayed in Fig.5 is listed in Table 1. The RMS error of the entire data set is $2.1 \%$, although the RMS error of the subset of data with $\mathrm{CT} \leq 40 \mathrm{y}$ is only $1.5 \%$, while the RMS error of the complementary set of data with $\mathrm{CT}=80 \mathrm{y}$ is $3.4 \%$.
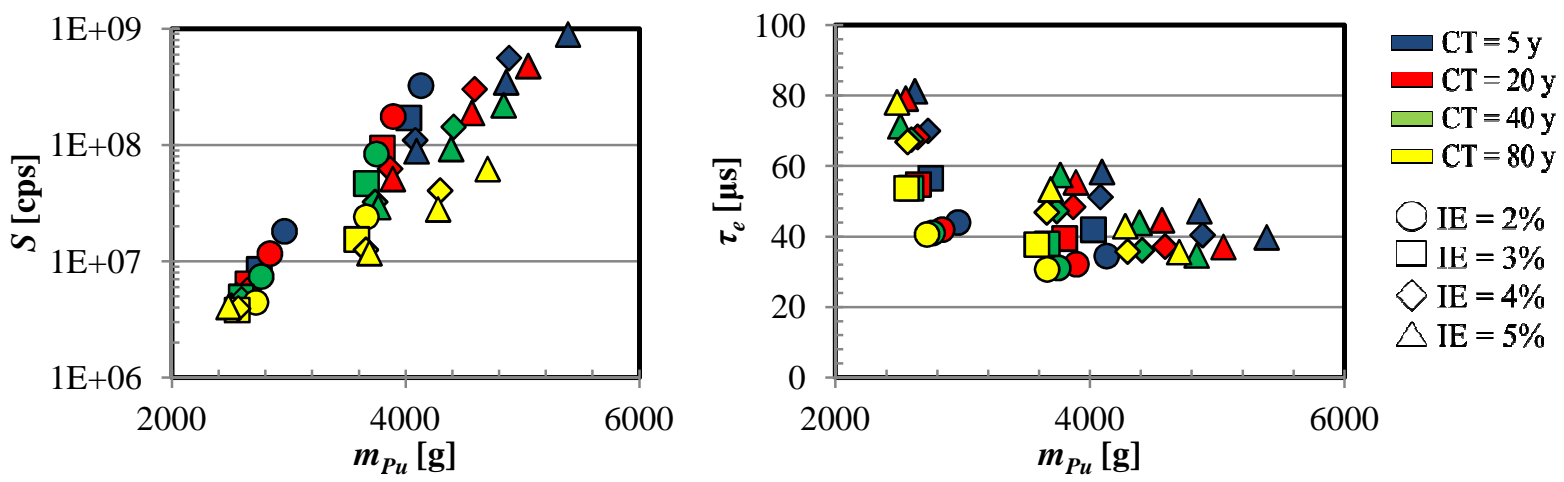

Fig. 4. Results of the simulations of the detected singles rate $S$ and $\tau_{e}$ for 44 SFAs from SFL2a as measured by the DDSI instrument. 

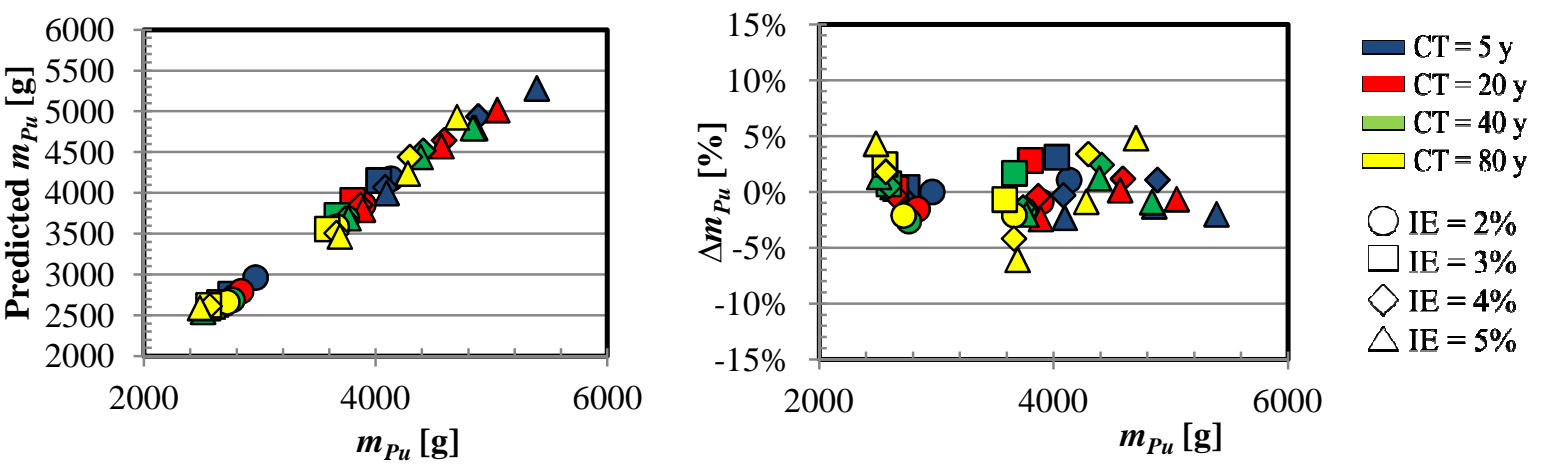

Fig. 5. Comparison of the absolute values of the $m_{P u}$ determined from eq. (6) and the true $m_{P u}$ in the SFA (left) and the relative differences between the determined $m_{P u}$ and the true values (right)

Table I. Summary of true and determined $m_{P u}$ for 44 SFAs from SFL2a divided into subsets by different CT values.

\begin{tabular}{|c|c|c|c|c|c|c|c|c|c|}
\hline $\begin{array}{c}\text { BU } \\
{[\mathbf{G W d}} \\
/ \mathbf{t U}]\end{array}$ & $\begin{array}{c}\text { IE } \\
{[\%]}\end{array}$ & $\begin{array}{l}\text { true } m_{P u} \\
{[\mathrm{~g}]}\end{array}$ & $\begin{array}{l}\text { det. } m_{P u} \\
{[\mathrm{~g}]}\end{array}$ & $\begin{array}{l}\Delta m_{P u} \\
{[\%]}\end{array}$ & $\begin{array}{c}\mathbf{B U} \\
{[\mathbf{G W d}} \\
/ \mathbf{t U}]\end{array}$ & $\begin{array}{c}\text { IE } \\
{[\%]}\end{array}$ & $\begin{array}{l}\text { true } m_{P u} \\
\quad[\mathrm{~g}]\end{array}$ & $\begin{array}{c}\text { det. } m_{P u} \\
{[\mathrm{~g}]}\end{array}$ & $\begin{array}{l}\Delta m_{P u} \\
{[\%]}\end{array}$ \\
\hline \multicolumn{5}{|c|}{$\mathrm{CT}=5 \mathrm{y}$} & \multicolumn{5}{|c|}{$\mathrm{CT}=20 \mathrm{y}$} \\
\hline 15 & 2 & 2961.5 & 2961.6 & $0.00 \%$ & 15 & 2 & 2836.6 & 2793.5 & $-1.52 \%$ \\
\hline 15 & 3 & 2746.5 & 2757.9 & $0.42 \%$ & 15 & 3 & 2649.8 & 2657.8 & $0.30 \%$ \\
\hline 15 & 4 & 2725.4 & 2709.2 & $-0.59 \%$ & 15 & 4 & 2644.0 & 2636.2 & $-0.29 \%$ \\
\hline 15 & 5 & 2622.6 & 2634.5 & $0.45 \%$ & 15 & 5 & 2551.4 & 2590.5 & $1.53 \%$ \\
\hline 30 & 2 & 4130.1 & 4174.2 & $1.07 \%$ & 30 & 2 & 3892.1 & 3857.9 & $-0.88 \%$ \\
\hline 30 & 3 & 4025.3 & 4150.7 & $3.11 \%$ & 30 & 3 & 3799.4 & 3906.7 & $2.83 \%$ \\
\hline 30 & 4 & 4080.6 & 4068.6 & $-0.29 \%$ & 30 & 4 & 3866.3 & 3852.1 & $-0.37 \%$ \\
\hline 30 & 5 & 4092.9 & 3998.9 & $-2.30 \%$ & 30 & 5 & 3888.2 & 3794.2 & $-2.42 \%$ \\
\hline 45 & 4 & 4883.8 & 4936.7 & $1.08 \%$ & 45 & 4 & 4588.9 & 4643.4 & $1.19 \%$ \\
\hline 45 & 5 & 4858.1 & 4796.1 & $-1.28 \%$ & 45 & 5 & 4565.4 & 4573.2 & $0.17 \%$ \\
\hline 60 & 5 & 5388.0 & 5280.7 & $-1.99 \%$ & 60 & 5 & 5047.6 & 5015.4 & $-0.64 \%$ \\
\hline \multicolumn{4}{|c|}{ Standard Deviation: } & $1.5 \%$ & \multicolumn{4}{|c|}{ Standard Deviation: } & $1.4 \%$ \\
\hline \multicolumn{5}{|c|}{$\mathrm{CT}=40 \mathrm{y}$} & \multicolumn{5}{|c|}{$\mathrm{CT}=80 \mathrm{y}$} \\
\hline 15 & 2 & 2761.9 & 2690.1 & $-2.60 \%$ & 15 & 2 & 2717.7 & 2660.6 & $-2.10 \%$ \\
\hline 15 & 3 & 2591.9 & 2610.9 & $0.73 \%$ & 15 & 3 & 2557.3 & 2620.4 & $2.47 \%$ \\
\hline 15 & 4 & 2595.0 & 2608.6 & $0.53 \%$ & 15 & 4 & 2565.4 & 2611.8 & $1.81 \%$ \\
\hline 15 & 5 & 2508.6 & 2542.3 & $1.34 \%$ & 15 & 5 & 2482.4 & 2589.8 & $4.33 \%$ \\
\hline 30 & 2 & 3748.9 & 3688.2 & $-1.62 \%$ & 30 & 2 & 3662.0 & 3586.1 & $-2.07 \%$ \\
\hline 30 & 3 & 3663.3 & 3724.5 & $1.67 \%$ & 30 & 3 & 3580.8 & 3556.4 & $-0.68 \%$ \\
\hline 30 & 4 & 3737.2 & 3685.1 & $-1.40 \%$ & 30 & 4 & 3659.3 & 3506.7 & $-4.17 \%$ \\
\hline 30 & 5 & 3764.7 & 3688.9 & $-2.01 \%$ & 30 & 5 & 3689.8 & 3465.8 & $-6.07 \%$ \\
\hline 45 & 4 & 4408.9 & 4516.0 & $2.43 \%$ & 45 & 4 & 4294.2 & 4440.1 & $3.40 \%$ \\
\hline 45 & 5 & 4387.3 & 4441.5 & $1.24 \%$ & 45 & 5 & 4275.9 & 4238.5 & $-0.87 \%$ \\
\hline 60 & 5 & 4838.0 & 4794.2 & $-0.91 \%$ & 60 & 5 & 4700.3 & 4927.0 & $4.82 \%$ \\
\hline \multicolumn{4}{|c|}{ Standard Deviation: } & $1.6 \%$ & \multicolumn{4}{|c|}{ Standard Deviation: } & $3.4 \%$ \\
\hline
\end{tabular}
indicated in Fig. 6 should be considered with limitations, however the trend seems to be rather smooth raising the possibility of a straight forward calibration with more data. Comparing these trends to results 
previous results do not, as shown in Fig. 6. However, the CT of 1y that breaks the linear trend in the DDA results was not investigated in this work, therefore based on the range of CT investigated in both papers, we still can conclude that the CT dependence of the fitting parameters of eq. (6) is qualitatively very similar.
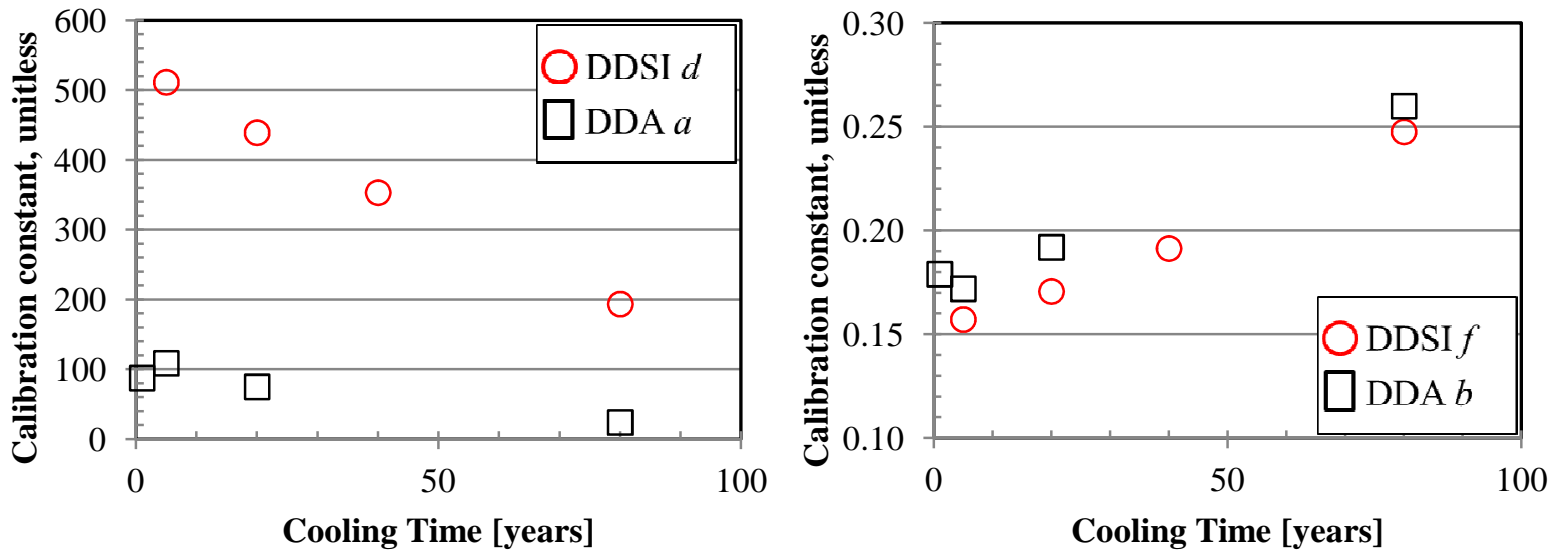

Fig.6 Values of the fitting parameters $d$ and from eq. (6) and a and $b$ from eq.(16) from [4] as the function of CT.

\section{CONCLUSIONS}

We have demonstrated through simulations that the recently developed approach of total plutonium determination based on measurement of SFA multiplication and passive neutron count rate is also applicable for use with the DDSI instrument. In the context of nondestructive assay of spent nuclear fuel by the DDSI instrument, the multiplication is measured in terms of the early die-away time of the Rossialpha distribution, and the passive neutron count rate is measured in the form of the detected singles rate. Overall, considering the CT known and discounting any systematic errors of real-life measurements, high fidelity simulations show that the total plutonium content of a SFA is determined with the RMS error of $2.1 \%$ when tested against 44 SFAs from SFL2a without explicit knowledge of the SFA initial enrichment or burnup.

\section{ACKNOWLDEGEMENTS}

This material is partially based upon work supported by the U.S. Department of Homeland Security under Grant Award Number, 2012-DN-130-NF0001-02. The authors also would like to acknowledge the support of the Next Generation Safeguards Initiative (NGSI), Office of Nonproliferation and International Security (NIS), National Nuclear Security Administration (NNSA). The views and conclusions contained in this document are those of the authors and should not be interpreted as necessarily representing the official policies, either expressed or implied, of the U.S. Department of Homeland Security or the National Nuclear Security Administration. LA-UR-13-29465. 


\section{REFERENCES}

1. M. A. Humphrey, S. J. Tobin, and K. D. Veal, "The Next Generation Safeguards Initiative's Spent Fuel Nondestructive Assay Project," Journal of Nuclear Materials Management, volume 40, No. 3, pp. 1-6, (2012).

2. H. O. Menlove, S. H. Menlove, S. J. Tobin, "Fissile and fertile nuclear material measurements using a new differential die-away self-interrogation technique", Nuclear Instruments and Methods in Physics Research A, volume 602, pp. 588-593. (2009)

3. A. C. Kaplan, V. Henzl, H. O. Menlove, M. T. Swinhoe, A. P. Belian, M. Flaska, S. A. Pozzi, "Determination of Spent Nuclear Fuel Assembly Multiplication with the Differential Die-Away Self-Interrogation Instrument", Nuclear Instruments and Methods A, volume 757, No. 1, pp. 2027, (2014).

4. V. Henzl, S. Croft, M. T. Swinhoe, S. J. Tobin, "Determination of the plutonium content in a spent fuel assembly by passive and active interrogation using a differential die-away instrument," Nuclear Instruments and Methods in Physics Research A, volume 712, pp. 83-92. (2013)

5. J. D. Galloway, H. R. Trellue, M. L. Fensin, and B. L. Broadhead, "Design and Description of the NGSI Spent Fuel Library with an Emphasis on Passive Gamma Signal," Journal of Nuclear Materials Management Vol.40, No.3, p25, (2012).

6. V. Henzl, M. T. Swinhoe, S. J. Tobin, and H. O. Menlove, "Measurement of the Multiplication of a Spent Fuel Assembly with the Differential Die-Away Method within the Scope of the Next Generation Safeguards Initiative Spent Fuel Project." JNMM-Journal of the Institute of Nuclear Materials Management 40.3 (2012).

7. T. D. Reilly, N. Ensslin, H. A. Smith, S. Kreiher (Eds.), Passive Nondestructive Assay of Nuclear Materials, NUREG/CR-5550 (1991).

8. Bignan, G. and Martin-Deidier, L. "Evaluation of the U-Pu Residual Mass from Spent Fuel Assemblies with Passive and Active Neutronic Methods," Proceedings of the 13th Annual ESARDA Meeting, Avignon, France, p311. (1991)

9. D. Pelowitz (ed.), "MCNPX User's Manual", Version 2.7.0, Los Alamos National Laboratory, LA-CP-11-00438, (2011).

10. H. R. Trellue, "Development of Monteburns: a Code that Links MCNP and ORIGEN2 in an Automated Fashion for Burnup Calculations," LA-13514-T, Los Alamos National Laboratory (1998). 